\title{
Development and characterization of a flux-pumped lumped element Josephson parametric amplifier
}

\author{
Martina Esposito ${ }^{1, *}$, Joseph Rahamim $^{1}$, Andrew Patterson $^{1}$, Matthias Mergenthaler ${ }^{1}$, James \\ Wills ${ }^{1}$, Giulio Campanaro ${ }^{1}$, Takahiro Tsunoda ${ }^{1}$, Peter Spring ${ }^{1}$, Sophia Sosnina ${ }^{1}$, Salha \\ Jebari $^{1}$, Kitty Ratter ${ }^{1}$, Giovanna Tancredi ${ }^{1}$, Brian Vlastakis ${ }^{1}$, and Peter Leek ${ }^{1}$ \\ ${ }^{1}$ Clarendon Laboratory, Department of Physics, University of Oxford, OX1 3PU, United Kingdom
}

\begin{abstract}
Josephson parametric amplification is a tool of paramount importance in circuit-QED especially for the quantum-noise-limited single-shot readout of superconducting qubits. We developed a Josephson parametric amplifier (JPA) based on a lumped-element LC resonator, in which the inductance L is composed by a geometric inductance and an array of 4 superconducting quantum interference devices (SQUIDs). We characterized the main figures of merit of the device, obtaining a $-3 \mathrm{~dB}$ bandwidth $B W=15 \mathrm{MHz}$ for a gain $G=21 \mathrm{~dB}$ and a $1 \mathrm{~dB}$ compression point $P_{1 \mathrm{~dB}}=-115 \mathrm{dBm}$. The obtained results are promising for the future use of such JPA as the first stage of amplification for single-shot readout of superconducting qubits.
\end{abstract}

\section{Introduction}

Josephson parametric amplification has received growing attention in the last decade in the field of circuit quantum electrodynamics (circuit-QED) [1-3] becoming a fundamental tool in superconducting quantum information research [4-14]. By parametrically modulating the inductance of one or more Josephson junctions, it is possible to amplify an incoming microwave signal by adding only the minimum amount of noise allowed by quantum mechanics. The major application of this is single-shot readout of superconducting qubits [15].

The mechanism of Josephson parametric amplification can be described in complete analogy to the one of optical parametric amplification, routinely used in laser laboratories $[16,17]$. In optics, the refractive index of a non-linear crystal is parametrically modulated in time with a pump field at frequency $\omega_{p}$ in order to amplify an input optical signal at frequency $\omega_{s}$, with the creation of an idler field at frequency $\omega_{i}$. Analogously, in superconducting circuits, the non-linear inductance of one or more Josephson junctions is parametrically modulated with a pump field in order to amplify an input signal in the microwave regime, by creating an additional idler field through a wave-mixing process.

The gain process in Josephson parametric amplification can be enhanced by using essentially two approaches; the first consists in embedding the Josephson non-linear elements in a microwave resonator which works in reflection and defines the bandwidth of operation of the amplifier (resonant-JPA) [4-11]; the second approach instead uses a very large number (typically hundreds) of Josephson elements in a long microwave coplanar wave guide working in

\footnotetext{
*e-mail: martina.esposito@physics.ox.ac.uk
} 
transmission, exploiting a long co-propagation of the pump field through the non-linear elements (Josephson travelling wave parametric amplifier, JTWPA) [14, 18]. Here we focused on the first approach that has the advantage to require a much simpler fabrication process and can potentially provide a broadband gain (hundreds of $\mathrm{MHz}$ ) if an appropriate impedance engineering is adopted [19].

Depending on the pumping scheme, resonant-JPAs can be further divided in two main categories that we could define current-pumped JPAs and flux-pumped JPAs [11, 20]. In currentpumped JPAs [4, 9, 21, 22], the AC pump signal is directly sent through the resonator, which includes one or more Josephson junctions, leading to a four-wave-mixing process $\left(2 \omega_{p}=\omega_{s}+\omega_{i}\right)$ due to the fact that the inductance $L$ is proportional to the square of the driving pump current [20]. On the other hand, in flux-pumped JPAs, one or more superconducting quantum interference devices (SQUIDs) are embedded in the resonator; in this case the inductance can be modulated by varying the magnetic flux threading the SQUID loops, generating this time a three-wave-mixing process $\left(\omega_{p}=\omega_{s}+\omega_{i}\right)$ because the inductance $\mathrm{L}$ is directly proportional to the driving current [20]. This last approach has been demonstrated in several labs $[6-8,10]$ and has the advantage that the pump signal doesn't travel through the resonator but it goes through a distinct flux-line, avoiding in this way any noise-contributions from the pump to the amplified signal.

We developed a flux-pumped JPA based on a well established design [10] developed in the Quantronics lab in Saclay, with the target to obtain $20 \mathrm{~dB}$ of gain and a minimum bandwidth of $10 \mathrm{MHz}$. The main difference from the Saclay design is the operational frequency range (our JPA is tunable in the 8-10 GHz range, while the one in [10] works in the 4-6 GHz range). These paremeters have been chosen in order to use the JPA, in future experiments, as first stage of amplification for the readout of a superconducting qubit based on a coaxial architecture recently developed in our lab in Oxford [23]. The basic unit of this architecture is made of a superconducting qubit and a linear resonator which are placed on the two opposite sides of a single chip, allowing out-of-plane readout and easy scalability to large arrays of qubits [23]. We configured the JPA design in order to obtain gain in the frequency range $(8-10 \mathrm{GHz})$ which correspond to the resonant frequency of our coaxial linear resonator [23]. In the following, the fabrication details and the characterization measurements of the developed JPA are shown.

\section{Circuit design and fabrication parameters}

The design of the JPA is shown in figure 1-(a). The LC resonator is made of a capacitance $C_{R}=275 \mathrm{fF}$, composed by two interdigitated capacitors, and an inductance $L$, composed by a meander of inductance $L_{g}=0.27 \mathrm{nH}$ and an array of 4 SQUIDs of inductance $L_{A}$. Finally a $50 \Omega$ coplanar wave guide is coupled to the resonator by an interdigitated coupling capacitor of capacitance $C_{C}=36 \mathrm{fF}$. The resonance frequency can thus be expressed as $f_{R}=1 / \sqrt{\left(L_{g}+L_{A}\right)\left(C_{C}+C_{R}\right)}$.

The device has been fabricated through a single-step electron beam lithography on a $5 \times 5 \times$ $0.5 \mathrm{~mm}$ sapphire chip, pattering the whole structure by evaporating a $100 \mathrm{~nm}$ aluminum layer. The SQUIDs, composed by a $8 \mu \mathrm{m} \times 11 \mu \mathrm{m}$ superconducting loop with two identical junctions with nominal area of $1.8 \mu \mathrm{m}^{2}$, have been fabricated with a double-angle evaporation technique. The measured room-temperature resistance of a single SQUID is $R_{\text {SQUID }}=100 \pm 10 \Omega$, from which we infer the single-junction critical current $I_{C}=2.7 \pm 0.1 \mu \mathrm{A}$.

Once the design of the LC resonator is fixed, the single-junction critical current remains the most important degree of freedom in the fabrication. Indeed, from $I_{C}$ one can calculate the Josephson inductance of a single junction $L_{J 1}=\Phi_{0} /\left(2 \pi I_{C}\right)$, where $\Phi_{0}$ is the magnetic flux quantum. 

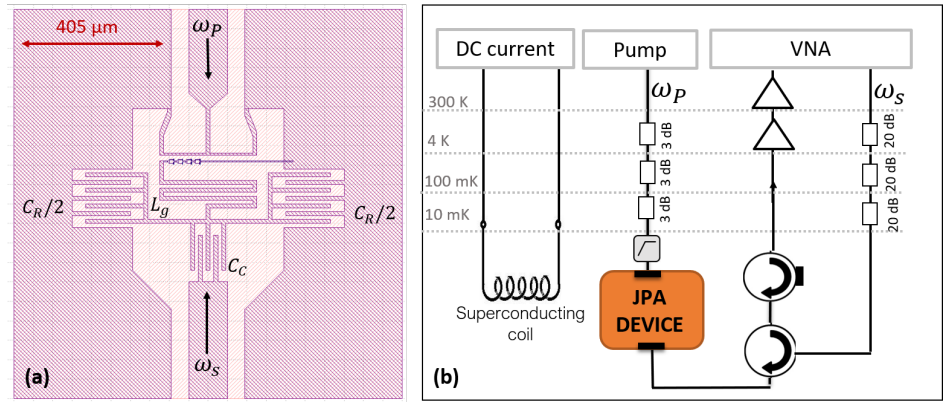

Figure 1. (a) CAD design of the JPA circuit. The coloured part indicates the aluminum layer and the white part indicates the sapphire substrate. A $50 \Omega$ coplanar wave guide, where the $\omega_{s}$ signal travels, is coupled to an LC resonator via a coupling capacitor $C_{c}$. The capacitance of the resonator is given by $C_{R}$ and the inductance is given by the geometric inductance $L_{g}$ and the Josephson inductance of an array of 4 SQUIDs shorted to ground. The pump signal, at frequency $\omega_{p}$ is sent through a flux line that is distant $15 \mu \mathrm{m}$ from the SQUIDs. (b) Schematic drawing of the cryogenic experimental set-up.

Once $L_{J 1}$ is known, it is possible to estimate two important parameters for the JPA. The first one is the single SQUID $\beta$ parameter [24], which takes into account the finite loop inductance of each SQUID and is defined as $\beta=L_{\text {loop }} / L_{J 1}$. It is desirable to keep this parameter low in order to avoid hysteresis problems of the SQUIDs [24, 25]. In our case, estimating the loop inductance as $1 \mathrm{nH}$ per $\mu m$, we obtain $\beta=0.30 \pm 0.01$.

The second important parameter is the so called participation ratio of the Josephson inductance $L_{J}$, which is defined as $p=L_{J} / L$, where $L$ is the total inductance. In detail, the Josephson inductance can be expressed as follows [10],

$$
L_{J}=\frac{N}{2} \frac{L_{J 1}}{\cos x+\frac{\beta}{2} \sin ^{2} x},
$$

where $\mathrm{N}$ indicates the number of SQUIDs and $x=\pi \Phi / \Phi_{0}$, with $\Phi$ the applied magnetic flux. On the other hand, the total inductance is,

$$
L=L_{g}+L_{A}, \quad \text { where } \quad L_{A}=\frac{\beta}{4} N L_{J 1}+L_{J}
$$

Using the above expressions (full derivation in [10]) we can estimate the participation ratio, obtaining in our case $p=L_{J} / L=0.44 \pm 0.1$. It is also desirable to keep the $p$ parameter low because it quantifies the Kerr non-linearity of the resonator [9], that, in flux-pumped JPA, should be kept low since it leads to saturation effects of the gain reducing the dynamic range [9-11]. It should be noticed that another way to decrease the Kerr non-linearity and increase the dynamic range of the amplifier is to increase the number of SQUIDs in the array $[9,10]$.

\section{JPA characterization measurements}

In this section we present the results of the JPA characterization measurements; in particular we show the characterization of the resonance frequency as a function of the DC flux, the gain-bandwidth characterization and the dynamic range characterization.

All the measurements have been performed using a dilution refrigerator at the base temperature of $10 \mathrm{mK}$. A schematic diagram of the experimental set-up is shown in figure 1-(b). 
The input signal is attenuated by $60 \mathrm{~dB}$ (see figure 1-(b)) and two circulators, working in the $8-12 \mathrm{GHz}$ range, are used to route the reflected microwave signal from the resonator to a cryogenic high electron mobility HEMT amplifier installed at the $4 \mathrm{~K}$ cryostat plate. The reflected signal is measured with a Vector Network Analyzer (VNA). The pump signal is attenuated by $9 \mathrm{~dB}$ and a high-pass filter is used (see figure 1-(b)). Finally, a superconducting coil is used to control the DC magnetic flux. The magnetic flux $\Phi$ threading the SQUIDs can be externally controlled in two ways, by sending current into the superconducting coil placed under the sample-holder thus generating a DC-flux, and by sending the pump time-dependent field through the flux-line on the chip by generating an AC-flux,

$$
\Phi=\Phi_{D C}+\Phi_{A C} \cos \left(\omega_{p} t+\chi\right)
$$

where $\omega_{p}$ is the pump frequency and $\chi$ a phase offset.

In figure 2 we show the measurement of the resonance frequency $f_{R}$ as a function of $\Phi_{D C}$, for $\Phi_{A C}=0$.
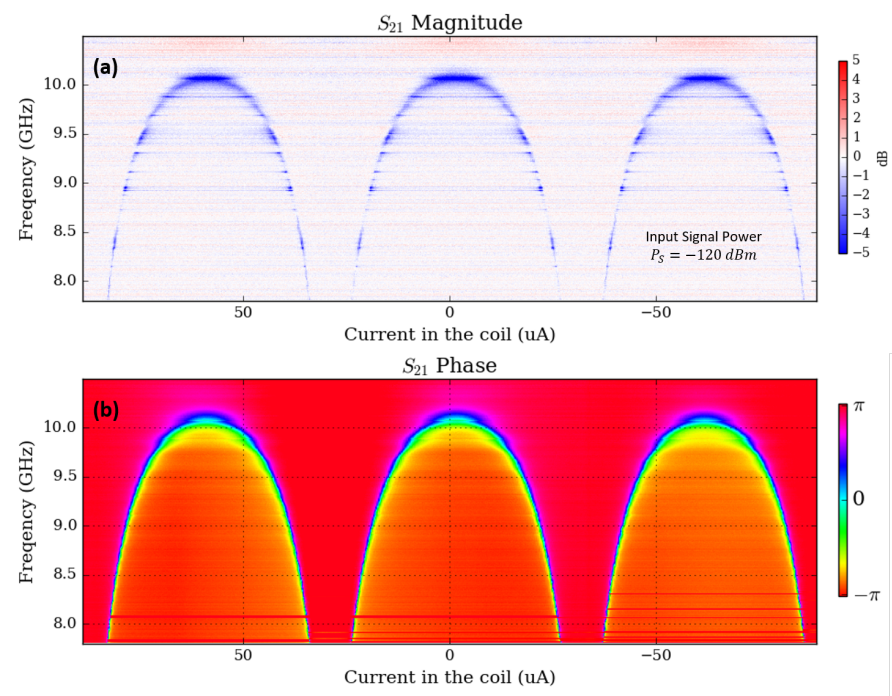

Figure 2. Magnitude (a) and phase (b) of the VNA scattering parameter $S_{21}$ for a reflection measurement of the JPA device as a function of the current sent into the superconducting coil (x-axis) and of input frequency (y-axis).

For each fixed current value we can estimate the resonance frequency $f_{R}$ and the quality factor $Q$ by fitting the phase data with a function $g(f)=-2 \arctan \left(2 Q\left(f / f_{R}-1\right)\right)$, where $f_{R}$ and $Q$ are free parameters. As an example, in figure 3 we report the data and the obtained best-fit function for $\Phi=\Phi_{0} / 4$. In this case we obtained a resonant frequency $f_{R} \approx 9.6$ and a quality factor $Q \approx 74$.

For a fixed $\Phi_{D C}$, it is possible to test the amplification of the system by switching on the pump signal at a frequency $f_{p}=2 f_{R}+\delta$ (where $f_{R}$ is the resonator frequency at that specific $\Phi_{D C}$ and $\delta$ is the detuning from the degenerate configuration) and gradually increasing the pump power. In figure 4 we show the gain obtained for different pump powers for a resonance frequency $f_{R} \approx 8.4 \mathrm{GHz}$. This measurement is obtained using an input signal power (at the sample) of $-120 \mathrm{dBm}$ and a small detuning $\delta=10 \mathrm{MHz}$ in order to avoid the degenerate regime $(\delta=0)$, in which the amplification is phase sensitive [10]. We observe a 


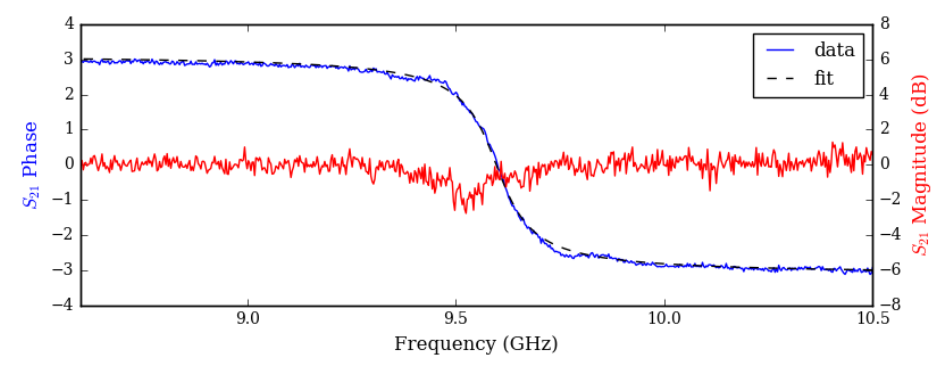

Figure 3. Magnitude and phase of the VNA scattering parameter $S_{21}$ for a reflection measurement of the JPA device for $\Phi=\Phi_{0} / 4$. The dashed line indicates the best-fit function for the phase data.

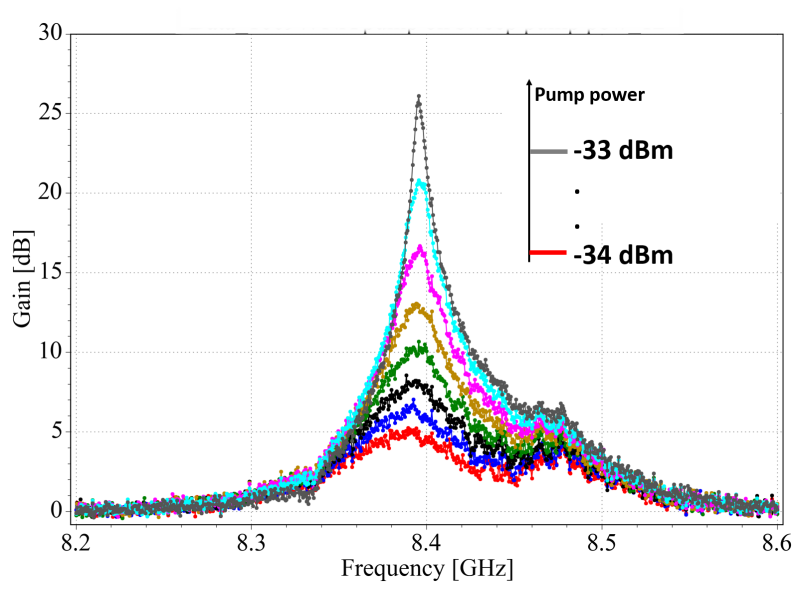

Figure 4. Gain obtained for different pump powers for a resonance frequency $f_{R} \approx 8.4 \mathrm{GHz}$. Different colors correspond to different input pump powers at the sample. The input signal power at the sample is $P_{s}=-20 \mathrm{dBm}$. The pump frequency is $f_{p}=2 f_{R}+\delta$, with $\delta=10 \mathrm{MHz}$

maximum gain $G=25 \mathrm{~dB}$ and we obtain a $-3 \mathrm{~dB}$ bandwidth $B W=15 \mathrm{MHz}$ for $G=21 \mathrm{~dB}$, which verifies our design target.

The characterization of the saturation power is shown in figure 5 . We obtain a $1 \mathrm{~dB}$ compression point, defined as the input signal power at which the gain decreases of $1 \mathrm{~dB}$, of $P_{1 \mathrm{~dB}}=-115 \mathrm{dBm}$ for $G=20 \mathrm{~dB}$. This result is comparable with the state of the art of flux-pumped JPAs using arrays of SQUIDs [10]. The results of the characterization measurements are very promising for the future use of the developed amplifier as first stage of amplification for the readout of superconducting qubits [23].

\section{Conclusions}

We have realized a flux-pumped JPA based on a lumped element LC resonator and an array of 4 SQUIDs. We characterized the amplifier performance obtaining a $-3 \mathrm{~dB}$ bandwidth $B W=15 \mathrm{MHz}$ for a gain $G=21 \mathrm{~dB}$ and a $1 \mathrm{~dB}$ compression point $P_{1 \mathrm{~dB}}=-115 \mathrm{dBm}$, 


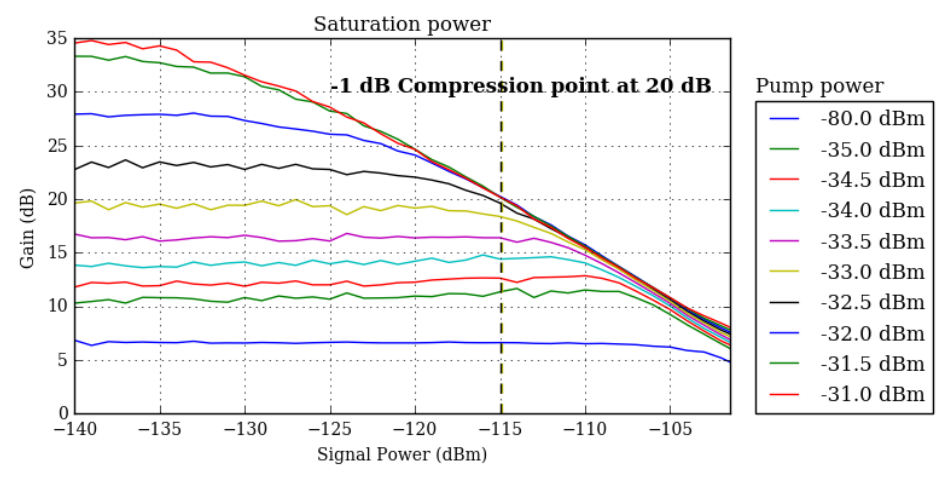

Figure 5. Saturation power characterization. Gain as a function of the input signal power at the sample. Different colors correspond to different input pump power at the sample. The dashed line indicates the value of the $1 \mathrm{~dB}$ compression point $P_{1 \mathrm{~dB}}=-115 \mathrm{dBm}$ for a gain of $20 \mathrm{~dB}$.

which are comparable with the state of the art of flux-pumped JPAs [10]. The estimation of the amplifier noise is subject of ongoing investigations. Overall, the performance of the amplifier are promising for our next goal which consists in using the developed amplifier as the first amplification stage for the single-shot readout of a coaxial transmon qubit, recently developed in our lab [23].

\section{References}

[1] R.J. Schoelkopf, S.M. Girvin, Nature 451, 664 EP (2008)

[2] M.H. Devoret, R.J. Schoelkopf, Science 339, 1169 (2013), http://science.sciencemag.org/content/339/6124/1169.full.pdf

[3] U. Vool, M. Devoret, International Journal of Circuit Theory and Applications 45, 897 (2017), https://onlinelibrary.wiley.com/doi/pdf/10.1002/cta. 2359

[4] M.A. Castellanos-Beltran, K.W. Lehnert, Applied Physics Letters 91, 083509 (2007), https://doi.org/10.1063/1.2773988

[5] V.E. Manucharyan, E. Boaknin, M. Metcalfe, R. Vijay, I. Siddiqi, M. Devoret, Phys. Rev. B 76, 014524 (2007)

[6] T. Yamamoto, K. Inomata, M. Watanabe, K. Matsuba, T. Miyazaki, W.D. Oliver, Y. Nakamura, J.S. Tsai, Applied Physics Letters 93, 042510 (2008), https://doi.org/10.1063/1.2964182

[7] L. Zhong, E.P. Menzel, R.D. Candia, P. Eder, M. Ihmig, A. Baust, M. Haeberlein, E. Hoffmann, K. Inomata, T. Yamamoto et al., New Journal of Physics 15, 125013 (2013)

[8] J.Y. Mutus, T.C. White, R. Barends, Y. Chen, Z. Chen, B. Chiaro, A. Dunsworth, E. Jeffrey, J. Kelly, A. Megrant et al., Applied Physics Letters 104, 263513 (2014), https://doi .org/10.1063/1.4886408

[9] C. Eichler, A. Wallraff, EPJ Quantum Technology 1, 2 (2014)

[10] X. Zhou, V. Schmitt, P. Bertet, D. Vion, W. Wustmann, V. Shumeiko, D. Esteve, Phys. Rev. B 89, 214517 (2014)

[11] A. Roy, M. Devoret, Phys. Rev. B 98, 045405 (2018) 
[12] M.A. Castellanos-Beltran, K.D. Irwin, G.C. Hilton, L.R. Vale, K.W. Lehnert, Nature Physics 4, 929 EP (2008)

[13] B. Ho Eom, P.K. Day, H.G. LeDuc, J. Zmuidzinas, Nature Physics 8, 623 EP (2012)

[14] C. Macklin, K. O'Brien, D. Hover, M.E. Schwartz, V. Bolkhovsky, X. Zhang, W.D. Oliver, I. Siddiqi, Science 350, 307 (2015), http://science.sciencemag.org/content/350/6258/307.full.pdf

[15] F. Mallet, F.R. Ong, A. Palacios-Laloy, F. Nguyen, P. Bertet, D. Vion, D. Esteve, Nature Physics 5, 791 EP (2009)

[16] E. Boyd, Non linear Optics (3rd ed.) (Academic Press, 2008)

[17] G. Cerullo, S. De Silvestri, Review of Scientific Instruments 74, 1 (2003), https://doi.org/10.1063/1.1523642

[18] K. O’Brien, C. Macklin, I. Siddiqi, X. Zhang, Phys. Rev. Lett. 113, 157001 (2014)

[19] T. Roy, S. Kundu, M. Chand, A.M. Vadiraj, A. Ranadive, N. Nehra, M.P. Patankar, J. Aumentado, A.A. Clerk, R. Vijay, Applied Physics Letters 107, 262601 (2015), https://doi.org/10.1063/1.4939148

[20] S. Boutin, D.M. Toyli, A.V. Venkatramani, A.W. Eddins, I. Siddiqi, A. Blais, Phys. Rev. Applied 8, 054030 (2017)

[21] M. Hatridge, R. Vijay, D.H. Slichter, J. Clarke, I. Siddiqi, Phys. Rev. B 83, 134501 (2011)

[22] L. Planat, R. Dassonneville, J.P. Martinez, F. Foroughi, O. Buisson, W. Hasch-Guichard, C. Naud, R. Vijay, K. Murch, N. Roch, Understanding the saturation power of josephson parametric amplifiers made from squids arrays (2018), arXiv: 1809.08476

[23] J. Rahamim, T. Behrle, M.J. Peterer, A. Patterson, P.A. Spring, T. Tsunoda, R. Manenti, G. Tancredi, P.J. Leek, Applied Physics Letters 110, 222602 (2017), https://doi.org/10.1063/1.4984299

[24] A. Barone, G. Patero, Physics and applications of the Josephson Effect (Wiley, 1982)

[25] S. Pogorzalek, K.G. Fedorov, L. Zhong, J. Goetz, F. Wulschner, M. Fischer, P. Eder, E. Xie, K. Inomata, T. Yamamoto et al., Phys. Rev. Applied 8, 024012 (2017) 